\title{
INCREASE IN SUBCELLULAR GSK-3 CLUSTERS IN INSULIN- AND ADRENALINE-TREATED DIFFERENTIATED RAT SKELETAL MUSCLE FIBRES
}

\author{
KATJA FinK $^{1 *}$, MATEJA Lobe PREBIL ${ }^{1 *}$, NinA VARDJAN ${ }^{1,2}$, JøRGEN JENSEN $^{3}$, \\ ROBERT ZOREC $^{\square, 1,2}$ AND MARKO KREFT ${ }^{凶, 1,2,4}$
}

${ }^{1}$ Laboratory of Neuroendocrinology-Molecular Cell Physiology, Institute of Pathophysiology, Faculty of Medicine, University of Ljubljana, Zaloška 4, Ljubljana, Slovenia, ${ }^{2}$ Celica Biomedical, Tehnološki park 24, Ljubljana, Slovenia, ${ }^{3}$ Department of Physical Performance, Norwegian School of Sport Sciences, Postboks 4014 Ullevål stadion, Oslo, Norway, ${ }^{4}$ Department of Biology, Biotechnical Faculty, University of Ljubljana, Ljubljana, Slovenia

*These authors have equally contributed to the work.

e-mail:katja.fink@mf.uni-lj.si,matejaprebil@gmail.com,nina.vardjan@mf.uni-lj.si,jorgen.jensen@nih.no, robert.zorec@mf.uni-lj.si,marko.kreft@mf.uni-lj.si

(Received February 25, 2020; revised March 9, 2020; accepted March 10, 2020)

\begin{abstract}
Glycogen synthase kinase 3 (GSK-3) plays an important role in metabolic regulation in skeletal muscles, and both insulin and adrenaline stimulate GSK-3 phosphorylation. The aim of the present study was to study the effect of insulin and adrenaline on GSK-3 localisation in skeletal muscles. We characterized subcellular localization of GSK-3 signal protein in fully differentiated muscle fibre by immunofluorescence and confocal microscopy. We stimulated muscle fibres with insulin and/or adrenaline. Images were analysed by segmentation of single central optical section of the muscle. We found GSK-3 to be localised in clusters. The number of GSK-3 clusters and their average size were increased after stimulation with insulin and/or adrenaline. Average GSK-3 particle size is linearly related to their quantity. We conclude that subcellular GSK-3 in isolated skeletal muscle fibres is localized in clusters and clustering increased after stimulation with insulin and/or adrenaline.
\end{abstract}

Keywords: adrenaline, GSK-3 (glycogen synthase kinase 3), insulin, skeletal muscle fibre.

\section{INTRODUCTION}

Skeletal muscles play a key role in regulating the whole body glucose metabolism. During insulin stimulation, more than $80 \%$ of the glucose disposal occurs in skeletal muscles where glucose is incorporated into glycogen, as shown by hyperglycemic-hyperinsulinemic clamp studies (Shulman et al., 1990). Note that disposition in skeletal muscle of $75-\mathrm{g}$ of ingested glucose in nondiabetic subjects is found to be $22 \%$ (Meyer et al., 2004). Insulin increases the rate of glucose uptake into the skeletal muscle and activates glycogen synthase (GS) (Shepherd et al., 1998, Chiang et al., 2003, Sano et al., 2003, Chowdhury et al., 2005, Mancinelli et al., 2017). Insulin resistance in type 2 diabetes is an outcome of several deficiencies in signal transduction, which is proposed to start with a discrepancy in insulin action on receptor tissue, such as skeletal muscle, and then loss of function in $\beta$-cells because of hyperinsulinemia (DeFronzo and Tripathy,
2009). The molecular targets and intracellular signalling systems that are modified during insulin resistance have received great attention in the past (Pessin and Saltiel, 2000, Björnholm and Zierath, 2005).

Insulin binds to its receptor and causes tyrosine phosphorylation of the insulin receptor substrate-1 (IRS-1) (White, 1998). Tyrosine phosphorylated IRS-1 binds and activates class-IA PI 3-kinase, a lipid kinase which phosphorylates phosphatidylinositol 4,5bisphosphate (PIP2) to phosphatidylinositol 3,4,5trisphosphate $\left(\mathrm{PIP}_{3}\right)$. When $\mathrm{PIP}_{3}$ is present in the membrane, protein kinase $\mathrm{B}$ (PKB) becomes activated by phosphorylation. Activated PKB phosphorylates and inactivates glycogen synthase kinase 3 (GSK-3), which is a major protein kinase involved in the regulation of glucose metabolism occurring in two known isoforms GSK-3 $\alpha$ and GSK-3 $\beta$ (Medina and Castro, 2008). Inactivated GSK-3 results in less phosphorylated and 
thus more active GS (Cross et al., 1995, Lawrence and Roach, 1997, McManus et al., 2005). In addition is GS regulated by allosteric factors, primarily by glucose 6 phosphate (Jensen \& Lai, 2009).

Inactivation of GSK-3 by phosphorylation has revealed GSK-3 ability to allow glycogen synthesis (Bouskila et al., 2008). GSK-3 is also involved in numerous cellular processes, including cell growth, proliferation, and differentiation (Cohen and Frame, 2001, Neary and Kang, 2006). GSK-3 is constitutively active, and multiple regulatory mechanisms affect GSK-3 inhibition in mammals (Cohen and Frame, 2001). The mechanism of these inhibitory signals affecting GSK-3 $\alpha$ and GSK-3 $\beta$ specifically are not known (Tanji et al., 2002).

Adrenaline has well characterised effects in muscle, a generally regarded counter-regulator of the insulin action. Adrenaline and insulin were believed to act via distinct pathways and the complex interactions between $\beta$-adrenergic and insulin signalling in skeletal muscles is not well understood (Brennesvik et al., 2005, Jensen et al., 2008). The individual responses generated by insulin and adrenaline do not predict the responses mediated by combined stimulation with these agonists (Jensen et al., 2007). Interestingly, adrenaline also regulates GSK-3 phosphorylation, but still inactivates GS activity (Jensen et al., 2008).

The aim of this study is to characterise the intracellular distribution and cluster formation of GSK3 in isolated skeletal muscle cells before and after stimulation with insulin and/or adrenaline. The results revealed that both hormones increase GSK-3 cluster size as well as their quantity in insulin- and adrenalinetreated skeletal muscle fibres.

\section{MATERIAL AND METHODS}

\section{EXPERIMENTAL ANIMALS}

Adult Wistar rats were used for the study. The animals were euthanized with carbon dioxide in accordance to the following ethical codes and directives: International Guiding Principles for Biomedical Research Involving Animals developed by the Council for International Organizations of Medical Sciences, and the Animal Protection Act (Official Gazette RS, No. 38/13). The experimental protocol was approved by The Administration of the Republic of Slovenia for Food Safety, Veterinary and Plant Protection (Republic of Slovenia, Ministry of
Agriculture, Forestry and Food, Ljubljana), Document No. U34401-47/2014/7.

\section{EXPERIMENT ON ISOLATED MUSCLE FIBERS AND IMMUNOFLUORES- CENCE}

Single muscle fibres were obtained from the flexor digitorum brevis muscle as described previously (Bekoff and Betz, 1977, Zorec et al., 1992). Briefly, the muscles were dissected from the animal and placed in $35 \mathrm{~mm}$ culture dishes, in $2 \mathrm{ml}$ Eagle's Minimal Essential Medium (EMEM; Sigma) supplemented with horse serum ( $5 \%$ by volume; Sigma), penicillin (100 units $\mathrm{ml}^{-1}$; Sigma), streptomycin (100 $\mu \mathrm{g} \mathrm{ml}^{-1}$; Sigma) and $2 \mathrm{mM}$ L-glutamine (Sigma). To this medium, collagenase (GIBCO, USA) was added $\left(3 \mathrm{mg} \mathrm{ml}^{-1}\right)$. The muscles were incubated for 3 hours at $36^{\circ} \mathrm{C}$ in an air-5\% $\mathrm{CO}_{2}$ incubator at $95 \%$ humidity, washed in fresh EMEM and mechanically dissociated into single muscle fibres by repeated passages through Pasteur pipettes. The isolated muscle fibres were cultured overnight on $22 \mathrm{~mm}$ glass coverslips with $10 \mu \mathrm{g} / \mathrm{cm}^{2}$ collagen type I (Sigma) and $1 \mu \mathrm{g} / \mathrm{cm}^{2}$ laminin (Sigma). Two hours before experiments, cultures were transferred into cell culture medium without serum and antibiotics. For stimulation, insulin $(70 \mathrm{nM}$, Novo Nordisk A/S, Denmark) and/or adrenaline (1 $\mu \mathrm{M}$, Sigma, USA) was added to the cell culture medium for 15 minutes.

For immunofluorescence single muscle fibres were washed with PBS (phosphate-buffered saline) and then fixed for 15 minutes in 4\% paraformaldehyde in PBS. The following 10 minutes muscle fibres were kept in fixative containing $0.1 \%$ of Triton X-100 and then four times washed with PBS. Non-specific staining was reduced by incubating muscle fibres in 3\% BSA (bovine serum albumin) and 10\% normal goat serum in PBS. Muscle fibres were then incubated with primary antibodies for 2 hours at $37^{\circ} \mathrm{C}$. We used rabbit polyclonal primary antibodies (Chemicon International, USA) antibodies diluted 1:500 for labelling GSK-3. Muscle fibres were then washed and incubated in PBS containing Alexa Fluor 488- or Alexa Fluor 546labelled goat anti-rabbit (1:500) and 3\% BSA for 45 minutes. Cells were washed and mounted in SlowFade Kit (Invitrogen, CA, USA) as described previously (Vardjan et al., 2007). 


\section{CONFOCAL IMAGING AND IMAGE ANALYSIS}

Images of single muscle fibres were acquired on a Zeiss LSM 510 confocal microscope (Zeiss, Jena, Germany) with a plan-apochromatic oil immersion objective $(63 \times, 1.4 \mathrm{NA})$. The Alexa Fluor 546 was excited with the $543 \mathrm{~nm}$ line of $\mathrm{He} / \mathrm{Ne}$ laser and Alexa Fluor 488 was excited with the $488 \mathrm{~nm}$ line of Argon laser. The emission signal was filtered by a $560 \mathrm{~nm}$ or $505 \mathrm{~nm}$ longpass filter. Muscle fibres were randomly selected. A single optical section of cylindrically shaped cultured single muscle fibres were taken at the central plane. Pixel dimensions were $0.14 \mu \mathrm{m} \times 0.14$ $\mu \mathrm{m}$. Pinhole diameter was 1.4 Airy Units $(132 \mu \mathrm{m})$, which resulted in the apparent optical slice thickness of $1 \mu \mathrm{m}$. Since the mean diameter of particle profile was $0.50 \mu \mathrm{m}$, the diameter of the profile in the image is only marginally underestimated due to sectioning (Košmelj et al., 2001). For analysis, we were using programme ImageJ, where we used the "Watershed" process to allow for the characterisation of the GSK-3 particle profiles (representing clustering of the protein), i.e. the number and size of their profiles within the reference space. The rectangle region with the surface area of $403.6 \mu \mathrm{m}^{2}$ (Fig. 1, the reference space), placed on the image of a single muscle fibres were used for analysis. The relative surface area of pixels with the intensity above the threshold level of $50 \%$ was calculated.

\section{RESULTS}

\section{THE NUMBER OF GSK-3 PARTICLE PROFILES AND THEIR AVERAGE SIZE INCREASED WITH INSULIN AND/OR ADRENALIN STIMULATION}

To learn about the subcellular distribution of GSK3 in single skeletal muscle fibres under control condition and after treatment with insulin and/or adrenaline we used confocal microscopy to image optical sections of immunofluorescent staining of GSK-3 in dissociated cultured muscle fibres (Fig. 1). We found GSK-3 to be localised in clusters, which were evenly distributed within the muscle fibre. Quantitative analysis of images consisted of binarization at the threshold level $50 \%$. The region-ofinterest with the surface area of $403.6 \mu^{2}$ was analysed. The number of GSK-3 above-threshold particle profiles in control images was $68.00 \pm 8.85$ $(\mathrm{n}=25$ Fig. $2 \mathrm{a})$. In images of muscle fibres, treated with $70 \mathrm{nM}$ insulin, the number of GSK-3-positive particles was elevated to $141.87 \pm 13.36(n=30)$.
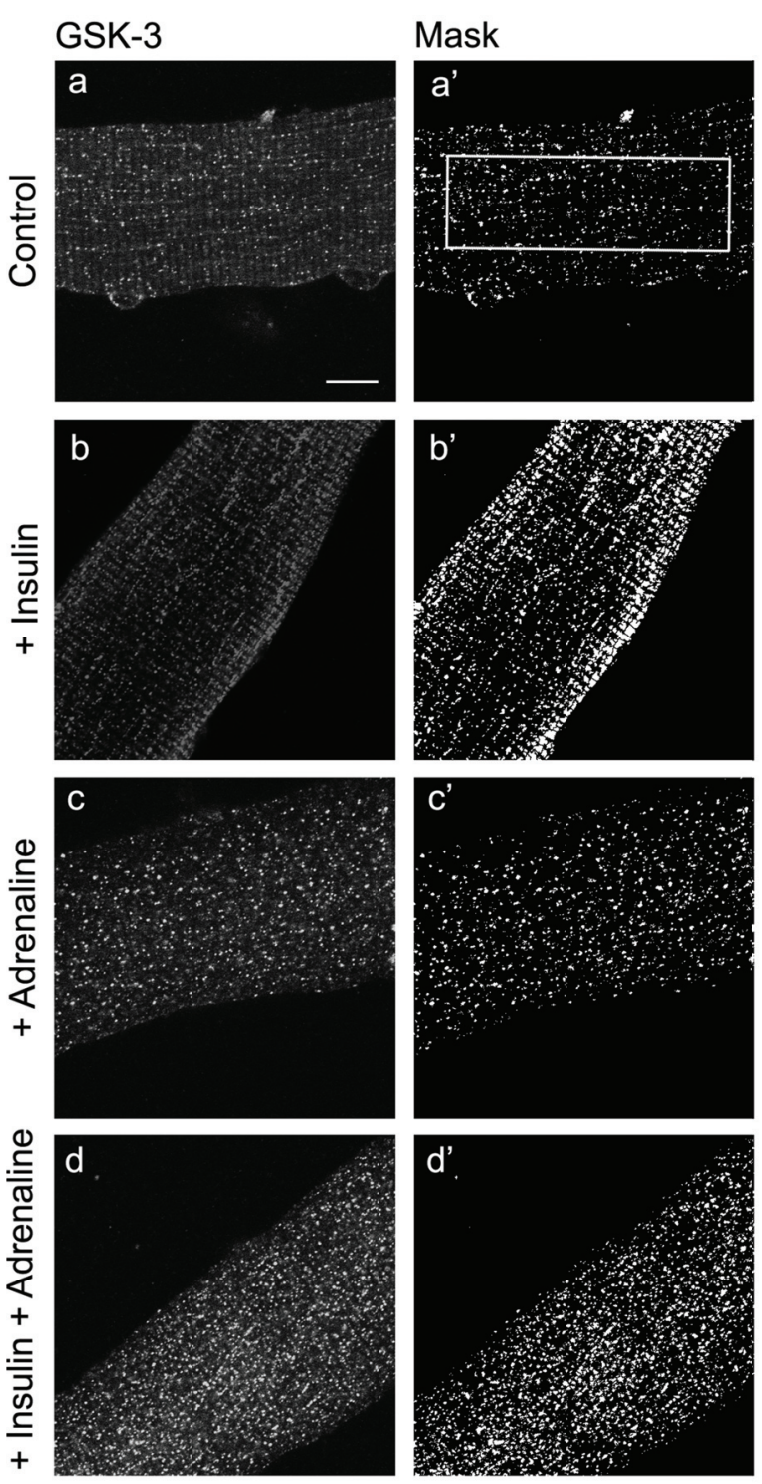

Fig. 1: Optical sections of immunofluorescent staining of GSK-3 in dissociated muscle fibres. The first panel shows a fibre with no stimulation (a, Control). Other fibres were stimulated with preincubation in insulin (b, +Insulin), adrenaline (c, +Adrenaline) or insulin and adrenaline $(d,+$ Insulin + Adrenaline $)$ as shown on the left panels. Right panels show images after thresholding the fluorescence signal (Mask) of the confocal sections (in Image J software, binarization at the threshold level $50 \%$ ). White square is depicting the region-of-interest with the surface area of $403.6 \mathrm{\mu m}^{2}$, which was used for analysis. Scale bar in a: $10 \mu \mathrm{m}$.

Similarly, following the treatment with $1 \mu \mathrm{M}$ adrenaline, the number of particle profiles was increased to $108.21 \pm 11.17(\mathrm{n}=29)$. The muscle fibres, which were stimulated with insulin and adrenaline simultaneously, showed 169.27 $\pm 13.96(\mathrm{n}=26)$ GSK-3 particle profiles. The average size of above-threshold 
GSK-3 particles in control fibres was: $0.21 \pm 0.01 \mu \mathrm{m}^{2}$ $(\mathrm{n}=25$; Fig. $2 \mathrm{~b})$, whereas in fibres treated with $70 \mathrm{nM}$ insulin the average size was $0.37 \pm 0.04 \mu \mathrm{m}^{2}(\mathrm{n}=30)$.

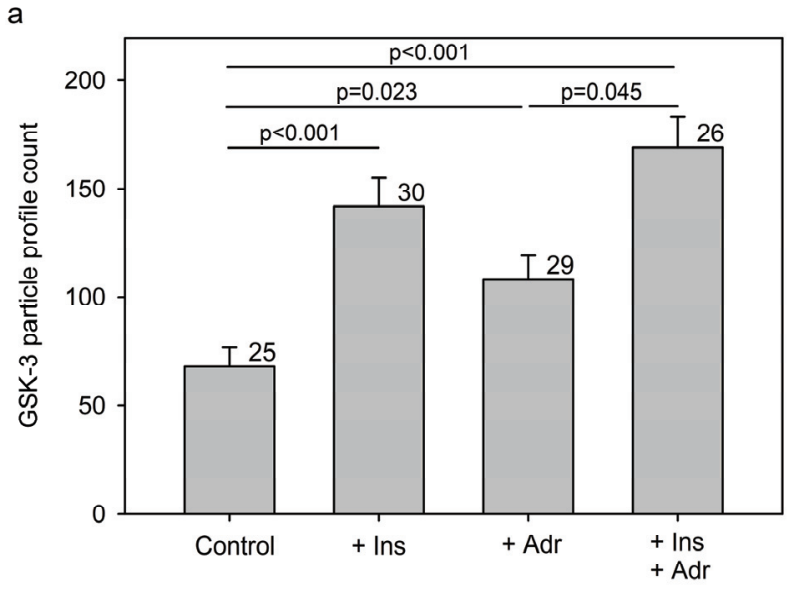

b

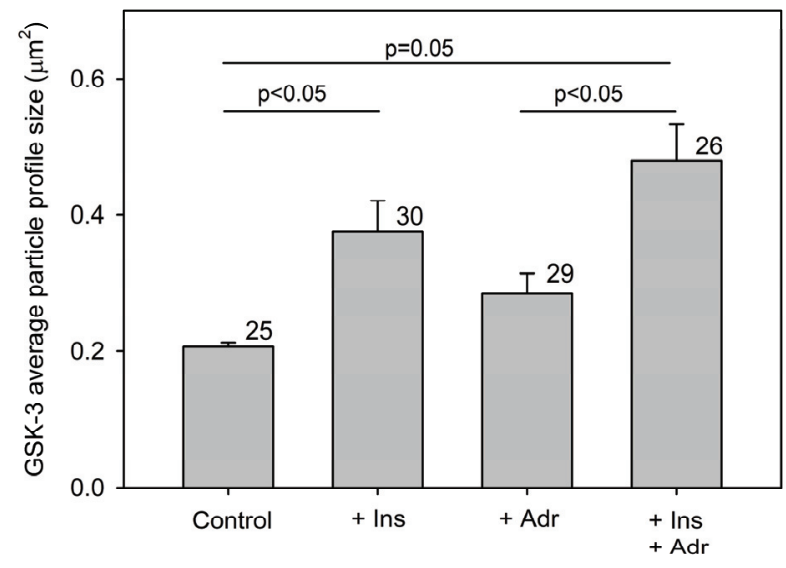

Fig. 2: The above-threshold area of GSK-3 positive signal in confocal images of control and stimulated isolated muscle fibres, measured as the number (a) of GSK-3 particle profiles and their average size (b). a) In images of control muscle fibres the number of GSK-3 particle profiles above intensity threshold level was 68 \pm 8.85 . In images of muscle fibres, treated with insulin $(+$ Ins; $70 \mathrm{nM})$, adrenaline $(+A d r ; 1 \mu M)$ or insulin and adrenaline $(+$ Ins + Adr $)$, the number of particle profiles were $141.87 \pm 13.36,108.21 \pm 11.17,169.27 \pm 13.96$, respectively. The insulin and/or adrenaline treatment significantly increases the GSK-3 positive area in isolated muscle fibres (p-value obtained by one-way ANOVA followed by all pairwise multiple comparison procedure Holm-Sidak method). b) In images of control muscle fibres, the average size of GSK-3 particle profiles (in $\mu^{2}$ ) was $0.21 \pm 0.01$. In images of muscle fibres, treated with insulin $(+$ Ins), adrenaline $(+$ Adr $)$ or insulin and adrenaline $(+\operatorname{Ins}+A d r)$, the relative area above the threshold level were $0.37 \pm 0.04,0.28 \pm$ $0.03,0.48 \pm 0.05$, respectively. The numbers by the bars indicate the number of analysed muscle fibres.
The insulin treatment and combined insulin/adrenaline treatment significantly increases the GSK-3 positive area in isolated muscle fibres (p-value obtained by Kruskal-Wallis one-way ANOVA on ranks followed by all pairwise multiple comparison procedure (HolmSidak method).

Similarly, when muscle fibres were treated with 1 $\mu \mathrm{M}$ adrenaline the average size of particle profiles was $0.28 \pm 0.03 \mu \mathrm{m}^{2}(\mathrm{n}=29)$ The muscle fibres, which were stimulated with insulin and adrenaline simultaneously, display the GSK-3 particle size of $0.48 \pm 0.05 \mu \mathrm{m}^{2}$ $(n=26)$. These results show a significantly increased (see below) clustering of GSK-3 in muscle fibres after stimulation with insulin and/or adrenaline, which is marked by the increased number of particle profiles as well as by the increased average size of GSK-3 particle.

We have performed One-way analysis of variance (ANOVA) test $(\mathrm{p}=0.001)$ for normally distributed data, followed by all pairwise multiple comparison procedure (Holm-Sidak method). We found the number of GSK-3 particle profiles in muscle fibres when stimulated with insulin $(p<0.001)$, adrenaline $(p=0.023)$ and combined insulin and adrenaline $(\mathrm{p}<0.001)$ are significantly different from control muscle fibres. Further, we have found that combined treatment with insulin and adrenaline has larger effect on particle count than treatment with adrenaline alone $(\mathrm{p}=0.045)$.

We have performed Kruskal-Wallis one-way ANOVA on ranks, where data was not normally distributed, followed by all pairwise multiple comparison procedure (Dunn's Method). We have found, that the average size of GSK-3 particle profiles in muscle fibres when stimulated with insulin $(\mathrm{p}=0.05)$, and combined insulin and adrenaline $(\mathrm{p}<0.05)$ are significantly different from control muscle fibres. Moreover, the average size of GSK-3 particle profiles in muscle fibres differ when stimulated with adrenaline alone vs. combined stimulation with insulin and adrenaline $(\mathrm{p}<0.05)$. We have found that combined treatment with insulin and adrenaline has larger effect on particle size than treatment with adrenaline alone $(\mathrm{p}<0.05)$.

\section{INSULIN AND/OR ADRENALINE STIMU- LATION OF MUSCLE FIBRE EXCITES CORRELATED INCREASE IN GSK-3 PARTICLE NUMBER AND SIZE}

We tested next whether the labelled GSK-3 particle number in a tested muscle fibre is correlated with the particle size. We found that larger GSK-3 particle appear only in muscle fibres with the number 
of particle profiles higher than 107 in the tested region of interest (Fig. 3). The latter coincides with the observation that the number of GSK-3 particle profiles in control muscle fibres is lower than this threshold.

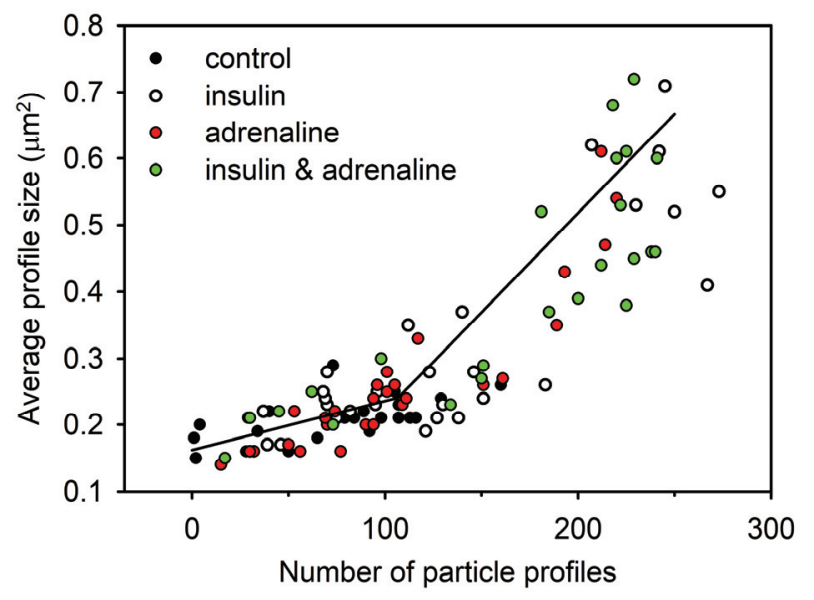

Fig. 3: Size of GSK-3 particle profiles is correlated to their number and is dependent on insulin and/or adrenaline stimulation. The average size of labelled GSK-3 particle is correlated $\left(r=0.719, p=4.3 \cdot 10^{-6}\right)$ to their number and the slope of linear regression is further increased after stimulation with insulin (white), adrenaline (red) or both (green). The broken regression line has its node at value 107 particle profiles per region of interest. At the node the average size of particle corresponds to $0.242 \mathrm{\mu m}^{2}$.

Consequently, their average size is largely limited to $0.35 \mu \mathrm{m}^{2}$. We found significant overall correlation between the number of particle profiles in a muscle fibre and the average particle size (Pearson correlation coefficient $r=0.719$, p: $4.3 \cdot 10^{-6}$ ). We fitted the brokenline regression to the data and found two slopes, first with parameters size (in $\left.\mu m^{2}\right)=0.162+0.0007 \pm$ $0.0002 \cdot N$ and second with parameters size (in $\left.\mu m^{2}\right)=$ $0.0761+0.0030 \pm 0.0005 \cdot N$, where $\mathrm{N}$ denotes the average number of particle profiles per region of interest $(p<0.0001)$. When stimulating muscle fibres with insulin and/or adrenaline, the number of GSK-3 particle profiles correlatively increases, which is evident from the increased regression slope on the broken line fit (Fig. 3). The broken regression line has its node at value 107 particle profiles per region of interest. At the node the average size of particle corresponds to $0.24 \mu \mathrm{m}^{2}$.

\section{DISCUSSION}

We characterised for the first time subcellular localisation of signal protein GSK-3 in fully differentiated muscle fibre by immunofluorescence and confocal microscopy. We report that clusters of GSK-3 exist in muscle without hormonal stimulation. Insulin and adrenaline are known to stimulate GSK-3 phosphorylation, which inactivates GSK-3 (Bouskila et al., 2008; Jensen et al., 2007), in our study increased the number and size of GSK-3 clusters in skeletal muscle.

Skeletal muscles are the specialised tissue for movement and the contractile proteins myosin and actin are localised in myofibrils surrounded by sarcoplasmic reticulum, mitochondria, glycogen particles, and other organelles (Nielsen and Ørtenblad, 2013), and it is unknown how signalling from surface receptors are transferred into the muscle fibres, which have diameters of $30 \mu \mathrm{m}$ or more. Our data show that GSK-3 exists in clusters that are homogenous distributed in the muscle fibres.

GSK-3 modulates the function of a diverse series of proteins, as well as being associated with a wide variety of human disorders, including neurodegenerative diseases, stroke, bipolar disorder, diabetes, and cancer. It controls microtubule and actin dynamics (Meijer et al., 2004, Zhou and Snider, 2005). Unlike many other kinases, GSK-3 $\beta$ is active in resting cells (Ciaraldi et al., 2007) and phosphorylation by PKB decreases GSK-3 activity. Interestingly, insulin which increases GSK-3 phosphorylation increased GSK-3 clustering. Although we did not use phosphorspecific antibodies to study localisation, it is tempting to speculate that it is the phosphorylated GSK-3 that accumulates in the clusters. Importantly, these clusters are localised throughout the muscle fibre. These data suggest that in unstimulated cell, the active GSK-3 molecules are diffusely distributed and phosphorylates target proteins. However, after GSK-3 inactivation by insulin stimulation, the inactive GSK-3 accumulates in clusters.

Adrenaline has similarly well characterized effects in muscle which via binding to $\beta$-adrenergic receptor, production of cAMP and activation of PKA results in breakdown of glycogen (Brennesvik et al., 2005). This mechanism results in raised insulin-sensitive glucose uptake in muscle (Kolnes et al., 2015). On the other hand, insulin dependent signalling phosphorylates GSK-3 and activates GS (Bouskila et al., 2008). Hence glycogen is essential in body glucose metabolism, specifically in skeletal muscle, where exercise induces insulin sensitization and enhances glucose uptake in part to enable glycogen supercompensation in the post exercise period (Hingst et al., 2018). This is due to increased sensitivity of muscle to insulin and higher GS 
activity during and after exercise (Hingst et al., 2018). Importantly, the individual responses generated by insulin and adrenaline do not predict the responses that will be generated by combined stimulation with these agonists (Jensen et al., 2007). It was reported previously that the adrenaline alone does not have any effect on the phosphorylation state or activity of PKB in skeletal muscle however, adrenaline potentiates insulin's effects on PKB (Brennesvik et al., 2005). Moreover, adrenaline alone stimulates GSK-3 Serine 9 phosphorylation via cAMP and PKA. Insulin and adrenaline are targeting distinct intracellular pools of PKB and GSK-3 suggesting that compartmentalization of different signalling molecules is likely to play an important role in dictating the responses that will be generated in muscle following stimulation with multiple agonists (Jensen et al., 2007).

Adrenaline induces phosphorylation of a pool of GSK-3 that is not involved in the regulation of glycogen metabolism (Brennesvik et al., 2005). The combination of adrenaline and insulin may activate novel signalling molecules rather than just summing up their effects on linear pathways. Adrenaline is a strong activator of PKB despite that it has no effect when insulin is absent (Jensen et al., 2008). Insulinstimulated glucose uptake increases following adrenaline despite prolonged exposure to hyperglycaemia in vivo. Increased insulin-stimulated glucose uptake and GS activation after adrenaline infusion cannot be explained by a reduction in glycogen content or an increase in PKB phosphorylation. Desensitization of $\beta$-adrenergic signalling pathway represents a potential mechanism for increased insulin action after adrenaline infusion (Jensen et al., 2005). Also, adrenaline does not influence basal or insulinstimulated PKB and GSK-3 $\beta$ phosphorylation in muscles, but completely blocks insulin-mediated GS activation and Serine 641 dephosphorylation. Still, insulin normalizes adrenaline-mediated hyperglycaemia (Jensen et al., 2010).

Insulin and adrenaline are regulating the PKB and GSK-3 in muscle, but the regulation is not uniform for the whole pool of molecules, but is having a specific effect on distinct pools of these signalling molecules (Jensen et al., 2007). Compartmentalisation can occur either via localisation to different organelles or by colocalisation of signalling molecules in specific complexes regulated by scaffold proteins. It has been shown, for example, that GSK-3 $\beta$ and PKA are both colocalised on AKAP-220 and that cAMP reduces the activity of GSK-3 in this complex to a greater degree than activity of the total cellular pool of GSK-3 and a protein phosphatase inhibitor inhibits the activity of GSK-3 $\beta$ bound to AKAP-220 more strongly than the total GSK-3 $\beta$ activity (Tanji et al., 2002, Jensen et al., 2007). AKAP-220, GSK-3 $\beta$, PKA, and type 1 protein phosphatase (PP1) were suggested to form a quaternary complex. PKA and PP1 regulate the activity of GSK$3 \beta$ efficiently by forming a complex with AKAP-220 (Tanji et al., 2002). It has become clear that scaffold proteins form complexes of signalling proteins to obtain specificity in signalling. Only a few PKBbinding proteins have been described (Brazil et al., 2002). Signalling seems to be ordered in modules and large protein complexes appear to direct signalling to organelles and regulate specific physiological functions for which software tool to analyse such complexes in fluorescent confocal images has been previously presented (Kreft et al., 2010). This is in line with our finding that GSK-3 is localised in skeletal muscle fibre in clusters (Fig. 1).

As discussed above, GSK-3 has complex regulation both upstream and downstream. In addition to insulin and adrenaline, Wnt signalling is well defined regulator of GSK-3 (Patel and Woodgett, 2017). It has been challenging to understand the complex interaction between insulin and adrenaline regulating GSK-3 and physiological processes, and the finding in the present study that GSK-3 clustering increases during hormonal stimulation that causes phosphorylation and inactivation will allow more in-depth studies of GSK-3 signalling. We did some attempts to study colocalisation of the GSK-3 cluster and PKB, which showed no co-localisation. Future studies should find organelles and proteins associated with the clusters. Furthermore, it will be important to confirm that GSK3 phosphorylation is the mechanism initiating GSK-3 cluster formation.

In conclusion, GSK-3 forms clusters in skeletal muscle fibres and clustering increases upon stimulation with insulin and adrenaline. This finding shows that it will be possible to dissect GSK-3 signalling in more detail in skeletal muscles. Furthermore, it will be important to characterise the physiological role of the cluster formation.

\section{ACKNOWLEDGEMENTS}

This work was supported by the grant \#P3 310 of Slovenian Research Agency. 


\section{CONFLICT OF INTEREST}

Authors declare no conflict of interests.

\section{ABBREVIATIONS}

AKAP-220 - A-Kinase Anchoring Protein 220; cAMP - cyclic adenosine monophosphate; GS glycogen synthase; GSK-3 -glycogen synthase kinase 3; IRS-1 - insulin receptor substrate-1; PIP3 phosphatidylinositol 3,4,5-trisphosphate; PKA protein kinase A; PKB - protein kinase B; PP1 - type 1 protein phosphatase; Wnt - wingless-related integration site signal transduction pathways.

\section{REFERENCES}

Bekoff A, Betz W (1977). Properties of isolated adult rat muscle fibres maintained in tissue culture. $\mathrm{J}$ Physiol 271:537-47.

Björnholm M, Zierath JR (2005). Insulin signal transduction in human skeletal muscle: Identifying the defects in type ii diabetes. Biochem Soc Trans 33:354-7.

Bouskila M, Hirshman MF, Jensen J, Goodyear LJ, Sakamoto K (2008). Insulin promotes glycogen synthesis in the absence of gsk3 phosphorylation in skeletal muscle. Am J Physiol Endocrinol Metab 294:E28-35.

Brazil DP, Park J, Hemmings BA (2002). Pkb binding proteins. Getting in on the akt. Cell 111:293-303.

Brennesvik E, Ktori C, Ruzzin J, Jebens E, Shepherd P, Jensen J (2005). Adrenaline potentiates insulinstimulated pkb activation via camp and epac: Implications for cross talk between insulin and adrenaline. Cell Signal 17:1551-9.

Chiang SH, Hwang J, Legendre M, Zhang M, Kimura A, Saltiel AR (2003). Tcgap, a multidomain rho gtpaseactivating protein involved in insulin-stimulated glucose transport. EMBO J 22:2679-91.

Chowdhury HH, Jevsek M, Kreft M, Mars T, Zorec R, Grubic Z (2005). Insulin-induced exocytosis in single, in vitro innervated human muscle fibres: A new approach. Pflugers Arch 450:131-5.

Ciaraldi TP, Nikoulina SE, Bandukwala RA, Carter L, Henry RR (2007). Role of glycogen synthase kinase-3 alpha in insulin action in cultured human skeletal muscle cells. Endocrinology 148:4393-9.

Cohen P, Frame S (2001). The renaissance of gsk3. Nat Rev Mol Cell Biol 2:769-76.
Cross DA, Alessi DR, Cohen P, Andjelkovich M, Hemmings BA (1995). Inhibition of glycogen synthase kinase- 3 by insulin mediated by protein kinase b. Nature 378:785-9.

DeFronzo RA, Tripathy D (2009). Skeletal muscle insulin resistance is the primary defect in type 2 diabetes. Diabetes Care 32 Suppl 2:S157-63.

Hingst JR, Bruhn L, Hansen MB, Rosschou MF, Birk JB, Fentz J, Foretz M, Viollet B, Sakamoto K, Faergeman NJ, Havelund JF, Parker BL et al., (2018). Exerciseinduced molecular mechanisms promoting glycogen supercompensation in human skeletal muscle. Mol Metab 16:24-34.

Jensen J, Ruzzin J, Jebens E, Brennesvik EO, Knardahl S (2005). Improved insulin-stimulated glucose uptake and glycogen synthase activation in rat skeletal muscles after adrenaline infusion: Role of glycogen content and pkb phosphorylation. Acta Physiol Scand 184:121-30.

Jensen J, Brennesvik EO, Lai YC, Shepherd PR (2007). Gsk-3beta regulation in skeletal muscles by adrenaline and insulin: Evidence that pka and pkb regulate different pools of gsk-3. Cell Signal 19:20410.

Jensen J, Grønning-Wang LM, Jebens E, Whitehead JP, Zorec R, Shepherd PR (2008). Adrenaline potentiates insulin-stimulated pkb activation in the rat fast-twitch epitrochlearis muscle without affecting irs-1associated pi 3-kinase activity. Pflugers Arch 456:969-78.

Jensen J, Lai YC (2009). Regulation of muscle glycogen synthase phosphorylation and kinetic properties by insulin, exercise, adrenaline and role in insulin resistance. Arch Physiol Biochem 115:13-21.

Jensen J, Ruge T, Lai YC, Svensson MK, Eriksson JW (2011). Effects of adrenaline on whole-body glucose metabolism and insulin-mediated regulation of glycogen synthase and pkb phosphorylation in human skeletal muscle. Metabolism 60:215-26.

Kolnes AJ, Birk JB, Eilertsen E, Stuenaes JT, Wojtaszewski JF, Jensen J (2015). Epinephrinestimulated glycogen breakdown activates glycogen synthase and increases insulin-stimulated glucose uptake in epitrochlearis muscles. Am J Physiol Endocrinol Metab 308:E231-40.

Košmelj K, Cedilnik A, Veranič P, Zupančič G, Rupnik M, Kocmur Bobanović L, Zorec R (2001). Intergranule fusion in rat pars intermedia cells. Image Anal Stereol 20:79-85.

Kreft M, Prebil M, Chowdhury HH, Grilc S, Jensen J, Zorec R (2010). Analysis of confocal images using variable-width line profiles. Protoplasma 246:73-80.

Lawrence JC, Roach PJ (1997). New insights into the role and mechanism of glycogen synthase activation by insulin. Diabetes 46:541-7. 
Mancinelli R, Carpino G, Petrungaro S, Mammola CL, Tomaipitinca L, Filippini A, Facchiano A, Ziparo E, Giampietri C (2017). Multifaceted roles of gsk-3 in cancer and autophagy-related diseases. Oxid Med Cell Longev 2017:4629495.

McManus EJ, Sakamoto K, Armit LJ, Ronaldson L, Shpiro N, Marquez R, Alessi DR (2005). Role that phosphorylation of gsk3 plays in insulin and wnt signalling defined by knockin analysis. EMBO J 20;24:1571-83.

Medina M, Castro A (2008). Glycogen synthase kinase-3 (gsk-3) inhibitors reach the clinic. Curr Opin Drug Discov Devel 11:533-43.

Meijer L, Flajolet M, Greengard P (2004). Pharmacological inhibitors of glycogen synthase kinase 3 . Trends Pharmacol Sci 25:471-80.

Meyer C, Woerle HJ, Dostou JM, Welle SL, Gerich JE. (2004). Abnormal renal, hepatic, and muscle glucose metabolism following glucose ingestion in type 2 diabetes. Am J Physiol Endocrinol Metab 287:E104956.

Neary J, Kang Y (2006). P2 purinergic receptors signal to glycogen synthase kinase-3beta in astrocytes. J Neurosci Res 84:515-24.

Nielsen J, Ørtenblad N (2013). Physiological aspects of the subcellular localization of glycogen in skeletal muscle. Appl Physiol Nutr Metab 38:91-9.

Nielsen JN, Richter EA. (2003). Regulation of glycogen synthase in skeletal muscle during exercise. Acta Physiol Scand 178:309-19.

Patel P, Woodgett JR (2017). Glycogen synthase kinase 3: A kinase for all pathways? Curr Top Dev Biol 123:277-302.
Pessin JE, Saltiel AR (2000). Signaling pathways in insulin action: Molecular targets of insulin resistance. J Clin Invest 106:165-9.

Sano H, Kane S, Sano E, Mîinea CP, Asara JM, Lane WS, Garner CW, Lienhard GE (2003). Insulinstimulated phosphorylation of a rab gtpase-activating protein regulates glut4 translocation. J Biol Chem 278:14599-602.

Shepherd PR, Withers DJ, Siddle K (1998). Phosphoinositide 3-kinase: The key switch mechanism in insulin signalling. Biochem J 333 ( Pt 3):47190 .

Shulman GI, Rothman DL, Jue T, Stein P, DeFronzo RA, Shulman RG (1990). Quantitation of muscle glycogen synthesis in normal subjects and subjects with noninsulin-dependent diabetes by $13 \mathrm{c}$ nuclear magnetic resonance spectroscopy. N Engl J Med 322:223-8.

Tanji C, Yamamoto H, Yorioka N, Kohno N, Kikuchi K, Kikuchi A (2002). A-kinase anchoring protein akap220 binds to glycogen synthase kinase-3beta (gsk-3beta ) and mediates protein kinase a-dependent inhibition of gsk-3beta. J Biol Chem 277:36955-61.

Vardjan N, Stenovec M, Jorgacevski J, Kreft M, Zorec R (2007). Subnanometer fusion pores in spontaneous exocytosis of peptidergic vesicles. J Neurosci 27:4737-46.

White MF (1998). The irs-signaling system: A network of docking proteins that mediate insulin and cytokine action. Recent Prog Horm Res 53:119-38.

Zhou FQ, Snider WD (2005). Cell biology. Gsk-3beta and microtubule assembly in axons. Science 308:2114.

Zorec R, Scuka M, Kordas M (1992). Effects of irreversible and reversible cholinesterase inhibitors on single acetylcholine-activated channels. J Membr Biol 125:41-8. 